\title{
All-optical switching using a new photonic crystal directional coupler
}

\author{
Babak Vakili ${ }^{1}$, Shahram Bahadori-Haghighi ${ }^{2}$, Rahim Ghayour ${ }^{3}$ \\ ${ }^{1,2,3}$ Department of Electrical and Computer Engineering, Shiraz University, Shiraz, Iran \\ E-mails: ${ }^{1}$ bvakili85@gmail.com, ${ }^{2}$ sbahadori.h@gmail.com, ${ }^{3}$ rghayour@shirazu.ac.ir \\ *corresponding author, E-mail: rghayour@shirazu.ac.ir
}

\begin{abstract}
In this paper all-optical switching in a new photonic crystal directional coupler is performed. The structure of the switch consists of a directional coupler and a separate path for a control signal called "control waveguide". In contrast to the former reported structures in which the directional couplers are made by removing a row of rods entirely, the directional coupler in our optical switch is constructed by two reduced-radius line-defect waveguides separated by the control waveguide. Furthermore, in our case the background material has the nonlinear Kerr property. Therefore, in the structure of this work, no frequency overlap occurs between the control waveguide mode and the directional coupler modes. It is shown that such a condition provides a very good isolation between the control and the probe signals at the output ports. In the control waveguide, nonlinear Kerr effect causes the required refractive index change by the presence of a high power control (pump) signal. Even and odd modes of the coupler are investigated by applying the distribution of the refractive index change in the nonlinear region of a super-cell so that a switching length of about 94 $\mu \mathrm{m}$ is obtained at the wavelength of $1.55 \mu \mathrm{m}$. Finally, alloptical switching of the $1.55 \mu \mathrm{m}$ probe signal using a control signal at the wavelength of $1.3 \mu \mathrm{m}$, is simulated through the finite-difference time-domain method, where both signals are desirable in optical communication systems. A very high extinction ratio of $67 \mathrm{~dB}$ is achieved and the temporal characteristics of the switch are demonstrated.
\end{abstract}

Keywords: All-optical switch, Nonlinear Kerr effect, Photonic crystal, Optical directional coupler

\section{Introduction}

In the past two decades, photonic crystal (PhC) emerges as a promising technology for optical integrated circuits. This structure is made of periodic dielectric materials in micron scale. PhC demonstrates unique optical properties, where the most interesting one is having adjustable photonic band gap $[1,2]$. On the other hand, slow light phenomenon that can be attained in $\mathrm{PhC}$ waveguides is known as a suitable approach to increase light-matter interactions [3-5]. By this approach, realization of ultra small nonlinear $\mathrm{PhC}$ devices is accessible [6-8]

A small scale all-optical switch in which the switching path of optical packets is controlled by another optical wave is a key component in recent optical systems [9]. In recent years many different structures have been proposed to implement all-optical switches. In almost all of the proposed structures the propagation properties of the devices are fine-tuned by using materials containing nonlinear optical properties $[10,11]$. Among the various nonlinear optical effects, Kerr effect can be used for all-optical switching operation.

Optical switches using directional couplers which are primarily proposed by Jensen [12] are based on optical nonlinearities. Hence, materials in which the nonlinear Kerr effect is strong enough are used in the waveguides of the coupler. Thus, by modulating the index of refraction of either the core or the cladding of the waveguides by a control signal, the propagation constants of the modes along the coupler can be modulated accordingly. Therefore, the coupling length and the power of the control signal have to be determined accurately to switch the optical signal path toward the appropriate output ports. So far, many all-optical switches using directional couplers including the $\mathrm{PhC}$ ones have been designed and demonstrated [13-15]. In fact, an optical switch based on $\mathrm{PhC}$ directional couplers comprising two parallel waveguides adjacent to each other is the most appropriate one. In this device, the required coupling length and control power for switching is reduced considerably due to the slow light effect.

Here in this paper, first the structure of the proposed $\mathrm{PhC}$ directional coupler for all-optical switching is introduced. The band structure of the switch with its corresponding control waveguide and directional coupler modes are calculated and shown to demonstrate the improvement obtained in this structure. In section 3 , the principles of the switching in such a structure are presented and the length of the switch is calculated through the analysis of the directional coupler modes. Finally in section 4, all-optical switching of the probe signal at the wavelength of $1.55 \mu \mathrm{m}$ using a high power pump signal at the wavelength of $1.3 \mu \mathrm{m}$ is performed and different characteristics of the switch including the high extinction ratio are investigated. 


\section{Proposed structure}

The schematic diagram of the all-optical switch is shown in Fig. 1. As it is seen, in addition to the $\mathrm{PhC}$ directional coupler, there is a separate path for control signal located between the waveguides of the directional coupler. In this path which is called "control waveguide", the nonlinear optical Kerr effect is applied [14].

In the proposed optical switch, as shown in Fig. 2, the PhC lattice arrangement is triangular of lattice constant $a$. It consists of rods with high refractive index of $n_{H}=3.5$ and radii of $0.2 a$ embedded in a background material with low refractive index of $n_{L}=1.3$. This structure has an optical band gap for TM modes within the normalized frequency range of $0.265<a / \lambda<0.386$, where $\lambda$ is the wavelength in free space.

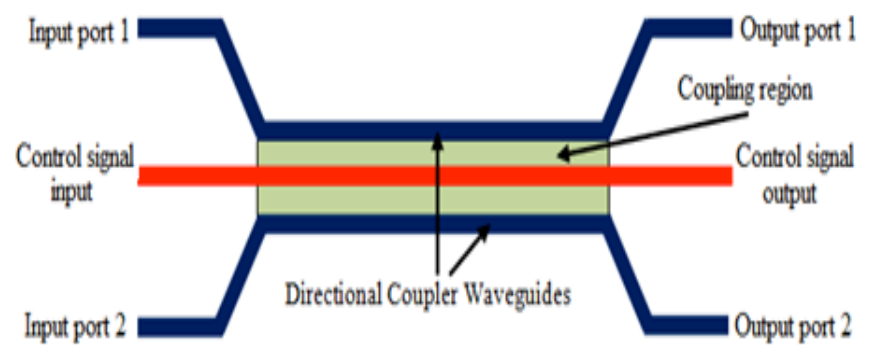

Figure 1: Schematic diagram of the all-optical switch based on a directional coupler.

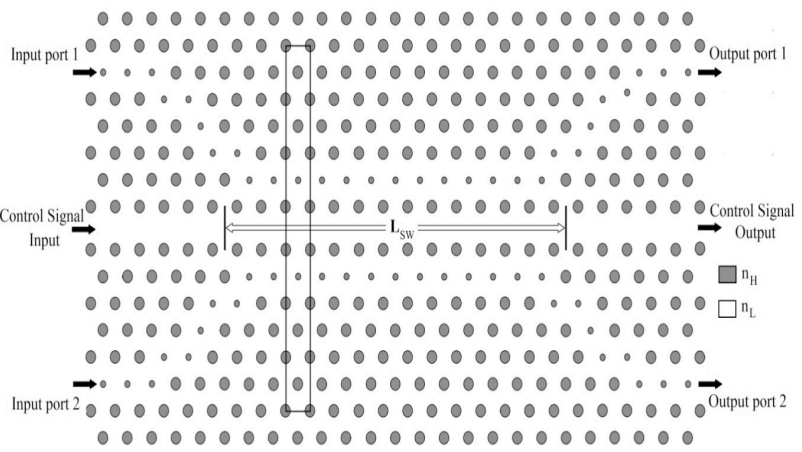

Figure 2: Structure of the proposed all-optical switch based on a PhC directional coupler.

Several methods are known to introduce and control the dispersion curves of guided modes in the photonic band gap of a $\mathrm{PhC}[16]$. In order to have a path for the control signal, a single-mode waveguide is made by removing a row of dielectric rods from the center of the structure as shown in Fig. 2. In addition, the width of this waveguide is reduced by moving the lower half of the structure upward and the upper half downward. By decreasing the width of the control waveguide, the dispersion curve of the guided mode shifts toward lower frequencies. Consequently, in the structure shown in Fig. 2, the control waveguide is constructed in such a way that a pump signal at the wavelength of $1.3 \mu \mathrm{m}$ is guided when its width is reduced to $0.8(a \sqrt{3})$. Afterward, the $\mathrm{PhC}$ directional coupler is created by setting two symmetric single-mode waveguides at the top and bottom of the control waveguide. Each waveguide of the coupler is constructed by reducing the radius of a row of dielectric rods to $0.1 a$, as shown in Fig. 2. However, in the previous structures reported in [13-15], the directional coupler is made by removing a row of rods entirely. Applying such a modification in this work provides the following advantages.

Fig. 3 shows the band structure of the proposed all-optical switch. As it is shown, there are three guided modes within the band gap including the control signal mode, odd and even directional coupler modes. The control signal mode has an even symmetry and is strongly confined within the control waveguide. Since there is no frequency overlap between the control waveguide mode and the directional coupler modes (see Fig. 3), it is guaranteed that the control signal cannot leak to the waveguides of the directional coupler. This is an improvement with respect to the old similar structures $[14,15]$. Therefore, a very good isolation between the optical signals at the output ports of the switch is expected. It is shown in the last section that by utilizing the proposed switch, a higher extinction ratio is achievable.

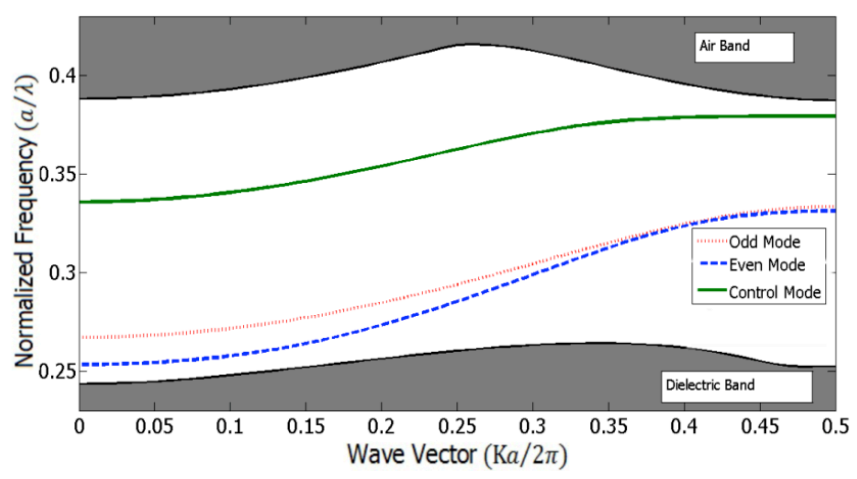

Figure 3: Band structure of the proposed all-optical switch shown in Fig. 2.

\section{Theory of switching}

A $2 \times 2$ directional coupler consists of two adjacent parallel waveguides and has two eigenmodes referred to as even and odd modes. The length of the adjacent waveguides at which the phase difference between these two modes are odd multiple of $\pi$, is called the coupling length and is obtained as follows:

$$
L_{C}=\frac{(2 n+1) \pi}{\left|k_{e}-k_{o}\right|}
$$

where $n$ is an integer number and $k_{e}$ and $k_{o}$ are wavenumbers of the even and odd modes, respectively. Similarly, if the phase difference between the two modes is even multiples of $\pi$ (i.e. $2 \mathrm{~m} \pi$ ) lightwave is fully coupled to the first waveguide again.

The refractive index of the nonlinear Kerr material, at the presence of an intensive optical signal, changes in proportion to the intensity of the optical field as follows: 


$$
n=n_{0}+n_{2} I
$$

where $n_{0}$ is the linear refractive index of the material, $n_{2}$ is the nonlinear Kerr coefficient and $I$ is the intensity of the applied optical field. Therefore, by using Kerr effect in the control waveguide the refractive index of the coupling region between the coupler waveguides will be modulated in proportion to the intensity of the control signal. Consequently, the dispersion curves of the even and odd modes are displaced. Hence, in the nonlinear case and at a specific frequency, wave-numbers of the odd and even modes are changed into $k_{o, n l i n}$ and $k_{e, \text { nlin }}$, respectively. For switching operation based on the directional coupler the following conditions must be satisfied [17]:

$$
\begin{gathered}
\left|k_{e, \text { lin }}-k_{o, l i n}\right| L_{S W}=(2 n+1) \pi \\
\left|k_{e, n l i n}-k_{o, n l i n}\right| L_{S W}=2 m \pi
\end{gathered}
$$

where $k_{e, \text { lin }}$ and $k_{o, \text { lin }}$ are wave-numbers of the even and odd modes in the linear case, respectively. $L_{S W}$ is the switching length as shown in Fig. 2. From Eq. (3) and Eq. (4), the switch length can be obtained as follows [17]:

$$
L_{S W}=\frac{(2 n+1) \pi}{\left|k_{e, \text { lin }}-k_{o, \text { lin }}\right|-\left|k_{e, \text { lin }}-k_{o, \text { nlin }}\right|}
$$

Fig. 4 shows the distribution of the refractive index change within the nonlinear control waveguide at the presence of the control signal. We determine the optical intensity of the control signal in such a way that maximum value of the refractive index change of $\Delta \mathrm{n}_{\max }=0.1$ at the center of the control waveguide is obtained. It is trivial that the refractive index changes are periodic over the entire length of the control waveguide with a periodicity of the lattice constant a.

The value of the lattice constant is taken as $a \cong 465 \mathrm{~nm}$ in order to set the operating point of $a / \lambda=0.3$ at $\lambda=1.55 \mu \mathrm{m}$. The Even and odd modes of the photonic crystal directional coupler around the wavelength of $1.55 \mu \mathrm{m}$ are shown in Figs. 5(a) and (b), respectively. Each figure shows both the linear and nonlinear cases of the corresponding modes. The dispersion curves of the modes in the nonlinear cases are achieved by introducing the refractive index distribution within the control waveguide shown in Fig. 4 in our calculation. Using the value of the wave-numbers shown in Figs. 5(a) and (b) and according to Eq. (5) the switching length of $L_{S W}=203 a$ at the wavelength of $1.55 \mu \mathrm{m}$ is obtained that is about $94 \mu \mathrm{m}$ for the considered value of the lattice constant.

At the end of this section, the estimated switching length vs. wavelength for different values of maximum refractive index changes in the control waveguide is also depicted in
Fig. 6. As can be seen, for larger values of refractive index changes, shorter switching lengths are required.

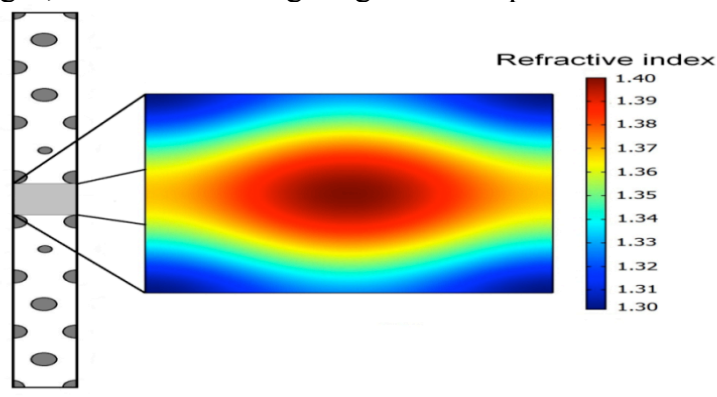

Figure 4: Distribution of the refractive index change within the control waveguide when an intense control signal is applied.

a

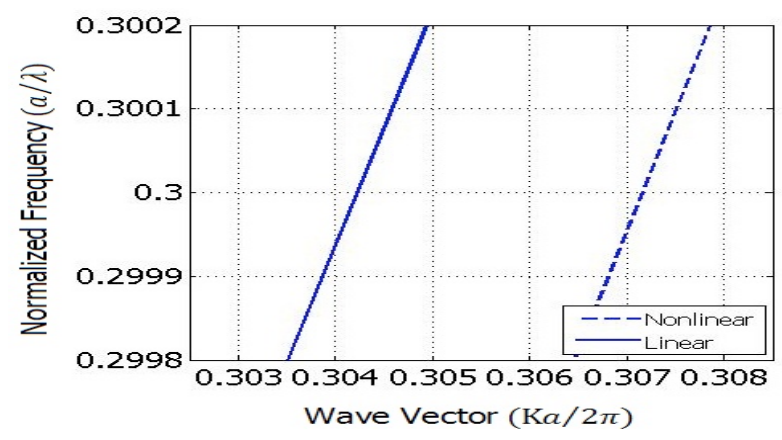

b

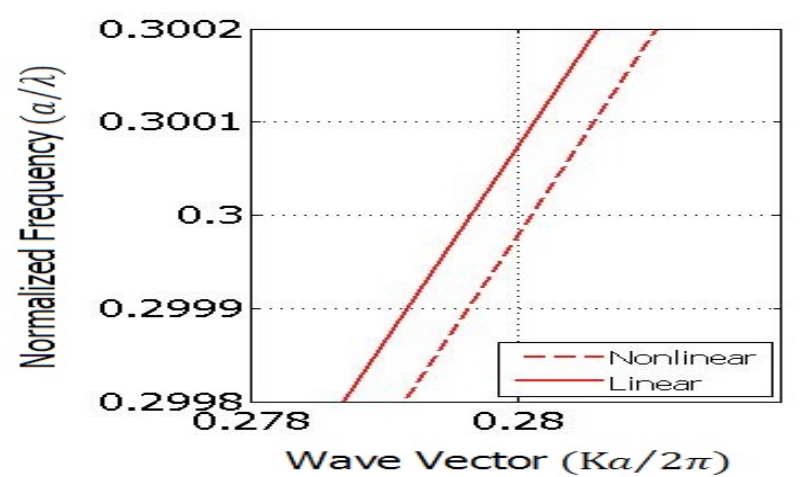

Figure 5: Dispersion curves of (a) the even and (b) the odd modes of the directional coupler for the linear and nonlinear cases.

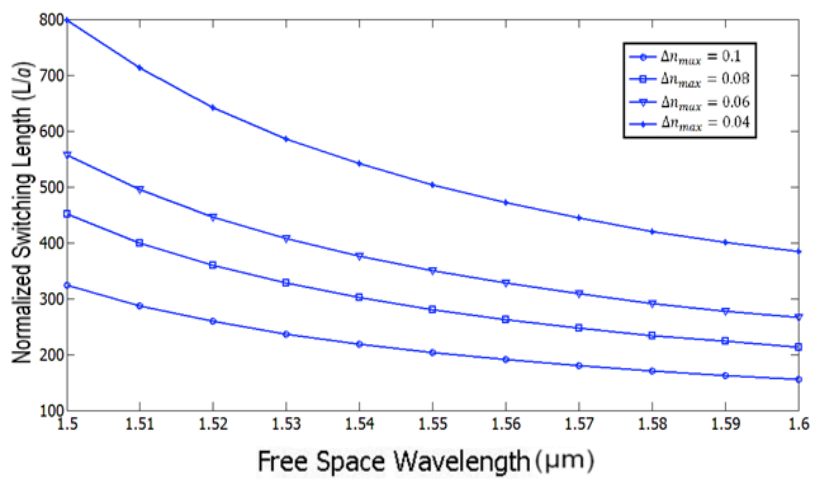

Figure 6: The relationship between the switching length of the $\mathrm{PhC}$ directional coupler and the wavelength for different values of maximum refractive index changes in the control waveguide. 


\section{Simulation results and discussion}

Now, in order to observe the operation of the proposed switch, the structure is simulated using finite-difference time-domain (FDTD) method along with fully absorbing layers at the borders to minimize reflections from the outer boundary of the computational cell. The resolution is set to $47 \times 47$ pixels $/ \mu \mathrm{m}^{2}$. First, the linear case of the switch in which no control signal is launched, is considered. As it is shown in Fig. 7(a), the input signal at the wavelength of 1.55 $\mu \mathrm{m}$ (probe signal) is transmitted to the output port 1 . In the nonlinear case, the control signal with the appropriate power and at the wavelength of $1.3 \mu \mathrm{m}$ is launched at the input of the control waveguide to induce the desired maximum refractive index change of 0.1 . As it is expected, the probe signal is directed to the output port 2 as shown in Fig. 7(b).

The transmitted powers to the output ports vs. wavelength for the linear and nonlinear cases around the wavelength of $1.55 \mu \mathrm{m}$ are shown in Figs. 8(a) and (b), respectively. It is seen that in both cases the power of the probe signal is mostly transmitted to the desirable output port of each case. From Figs. 8(a) and (b) an extinction ratio of $67 \mathrm{~dB}$ is calculated that is a higher value compared with the previous works $[15,18]$. It is also seen that the leakage of the power to the output of the control waveguide is negligible. For the sake of completeness, Fig. 8(c) is presented to show the isolation between the output ports around the wavelength of $1.3 \mu \mathrm{m}$ in the nonlinear case. In this case the control signal at the wavelength of $1.3 \mu \mathrm{m}$ is highly guided through the control waveguide so that there is a great isolation between the control waveguide and output ports 1 and 2 .

At this point, it should be mentioned that from a practical point of view there are some factors which result in radiation losses in our proposed optical switch. Generally, one of the main losses could be the one due to fabrication disorders. Disorders in photonic crystals cause scattering and reflections into the reverse-direction waveguide mode. Such losses have an inverse relationship with the group velocity of guided modes [19]. Therefore, disorder-induced losses are so considerable in slow light regimes of modes like near the band edges where the group velocities are very small. However, in our proposed structure the operating points are taken away from the band edges so that the group indices of even, odd and control modes are 3.57, 4.76 and 5.56, respectively. These values of group indices are much smaller than moderately slow light waveguides with group indices of approximately 20 [19]. Hence, significant disorderinduced losses will not be expected in our proposed directional coupler.

Finally in order to investigate the time-domain characteristics of the switch, the power of the output ports 1 and 2 vs. time are presented in Fig. 9. The control and the probe signals are turned on simultaneously at $t=0$ to put the switch in the nonlinear state. After $t=2$ ps the steady state is obtained in which the probe signal is highly guided to the output port 2 . At $t=3$ ps the control signal is turned off for the linear case. It is seen that at $t=5$ ps the probe signal starts to travel toward the output port 1 . The transition time after which the probe signal is completely coupled to the output port 1 is about 1 ps. a
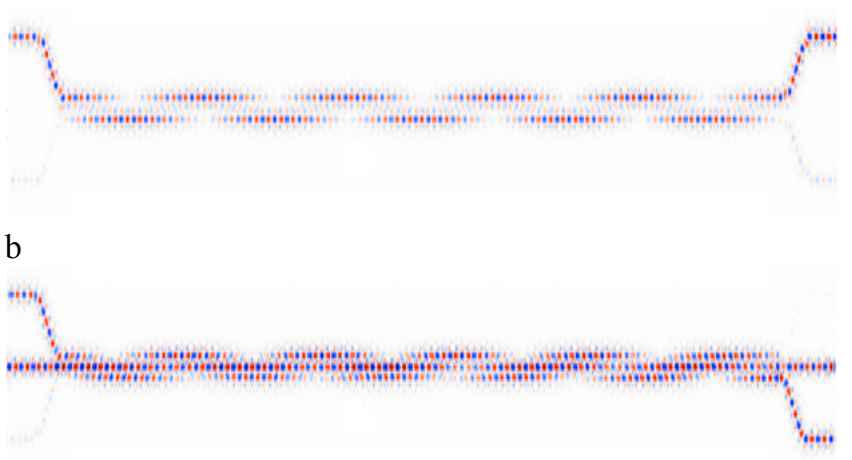

Figure 7: Snapshots of the probe signal at the wavelength of 1.55 $\mu \mathrm{m}$ propagating down the $\mathrm{PhC}$ directional coupler switch when (a) no control signal is launched (linear case) and (b) a control signal at the wavelength of $1.3 \mu \mathrm{m}$ is launched (nonlinear case).

a

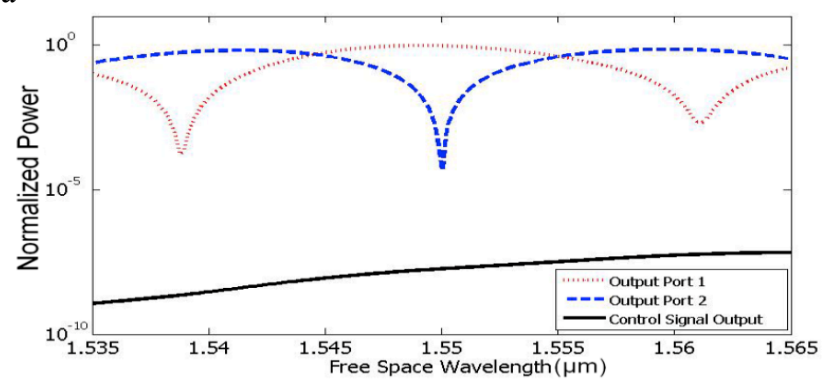

b

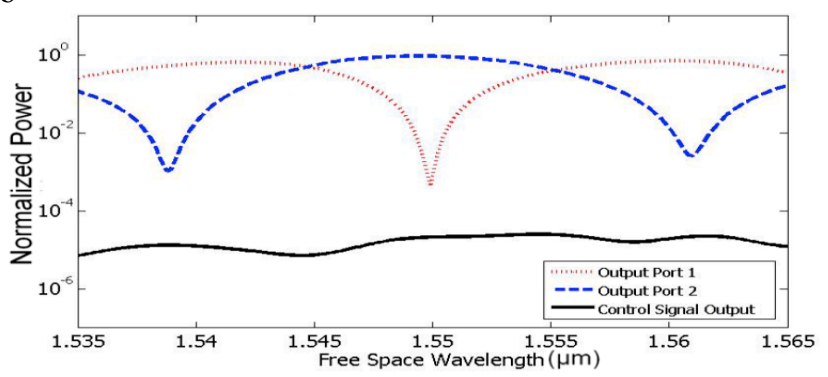

c

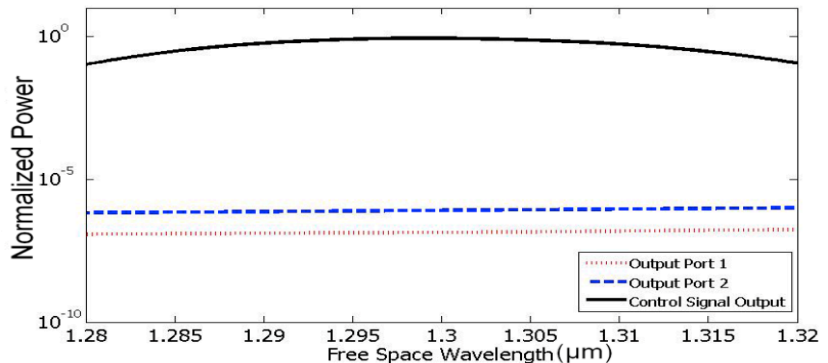

Figure 8: Transmitted power to the output ports of the PhC directional coupler for (a) the linear case, (b) the nonlinear case around $1.55 \mu \mathrm{m}$ and (c) the nonlinear case around $1.3 \mu \mathrm{m}$. 


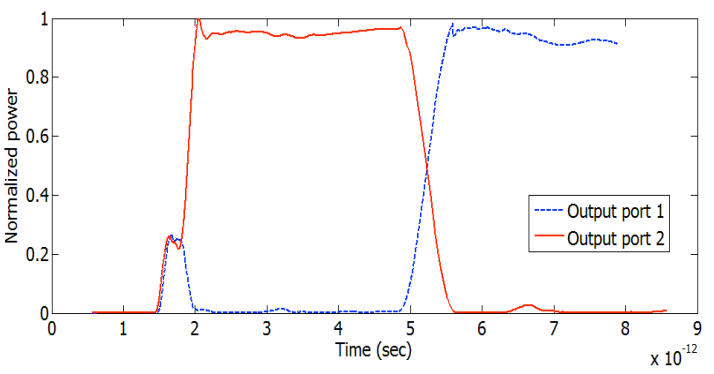

Figure 9: FDTD results of the $\mathrm{PhC}$ directional coupler to show its time-domain switching characteristics.

\section{Conclusions}

In summary, we proposed a new structure of $\mathrm{PhC}$ directional coupler for all-optical switching. After presenting the principles of the switching operation and calculating the even and modes for the linear and nonlinear cases, the required switching length of about $94 \mu \mathrm{m}$ at the wavelength of $1.55 \mu \mathrm{m}$ was obtained. The transient simulation of the alloptical switch using finite-difference time-domain method was performed and the corresponding time-domain characteristics were shown. A high extinction ratio of $67 \mathrm{~dB}$ was obtained by our proposed structure that is an improvement in comparison with the previous works. It was also demonstrated that no frequency overlap occurs between the control waveguide mode and the directional coupler modes in our proposed structure which results in a good isolation between the probe and control signals at the output ports. As another advantage, we performed the all-optical switching of a $1.55 \mu \mathrm{m}$ probe signal using a control signal with the wavelength of $1.3 \mu \mathrm{m}$. As it is known, both of these wavelengths are desirable in optical communication systems.

\section{References}

[1] J. D. Joannopoulos, S. G. Johnson, J. N. Winn, R. D. Meade, Photonic Crystals Molding the Flow of Light, Princeton University Press, 2008.

[2] W. Jiang, L. Gu, X. Chen, R. T. Chen, Photonic crystal waveguide modulators for silicon photonics: Device physics and some recent progress, Solid State Electron. 51: 1278-1286, 2007.

[3] T. F. krauss, Slow light in photonic crystal waveguides, J. Phys. D: Appl. Phys. 40: 2666-2670, 2007.

[4] Y. Chen, J. Mørk, Theory of carrier depletion and light amplification in active slow light photonic crystal waveguides, Opt. Express 21: 29392-29400, 2013.

[5] A. Khodamohammadi, H. Khoshsima, V. Fallahi, M. Sahrai, Wideband slab photonic crystal waveguides for slow light using differential optofluidic infiltration, Applied Optics 54: 1002-1009, 2015.

[6] S. Bahadori Haghighi, R. Ghayour, B. Vakili, Photonic crystal optical switch using a new slow light waveguide and heterostructure Y-junctions, Optik - Int. J. Light Electron Opt. 124: 6292-6297, 2013.
[7] Y. Xu, C. Caer, D. Gao, E. Cassan, X. Zhang, High efficiency asymmetric directional coupler for slow light slot photonic crystal waveguides, Opt. Express 22: 11021-11028, 2014.

[8] T. Baba, H. C. Nguyen, N. Yazawa, Y. Terada, S. Hashimoto, T. Watanabe, Slow-light Mach-Zehnder modulators based on Si photonic crystals, Sci. Technol. Adv. Mater. 15: 024602, 2014.

[9] T. Baba, Slow light in photonic crystals, Nat. Photonics 2: 465-473, 2008.

[10] P. Colman, Y. Yu, M. Heuck, P. Lunnemann Hansen, K. Yvind, J. Mørk, Temporal dynamics of alloptical switching in Photonic Crystal Cavity, CLEO: QELS_Fundamental Science, San Jose, California United States, 2014.

[11] F. Diebel, D. Leykam, M. Boguslawski, P. Rose, C. Denz, A. S. Desyatnikov, All-optical switching in optically induced nonlinear waveguide couplers, Appl. Phys. Lett. 104: 261111, 2014.

[12] S. Jensen, The nonlinear coherent coupler, IEEE J. Quantum Electron. 18: 1580-1583, 1982.

[13] A. Taher Rahmati, N. Granpayeh, Kerr nonlinear switch base on ultra-compact photonic crystal directional coupler, Optik - Int. J. Light Electron Opt. 122: 502-505, 2011.

[14]F. Cuesta-Soto, A. Martínez, B. García-Baños, J. Matrí, Numerical analysis of all-optical switching based on a 2-D nonlinear photonic crystal directional coupler, IEEE J. Sel. Topics Quantum Electron. 10: 1101-1106, 2004.

[15] M. Danaie, H. Kaatuzian, Improvement of power coupling in a nonlinear photonic crystal directional coupler switch, Photonics Nanostruct. Fundam. Appl. 9: 70-81, 2011.

[16] M. Lončar, J. Vučković, A. Scherer, Methods for controlling positions of guided modes of photoniccrystal waveguides, J. Opt. Soc. Am. B 18: 1362-1368, 2001.

[17] N. Yamamoto, T. Ogawa, K. Komori, Photonic crystal directional coupler switch with small switching length and wide bandwidth, Opt. Express 14: 1223-1229, 2006.

[18] A. T. Rahmati, N. Granpayeh, Design and simulation of a switch based on nonlinear directional coupler, Optik - Int. J. Light Electron Opt. 121: 1631-1634, 2010.

[19] L. O'Faolain, T. P. White, D. O’Brien, X. Yuan, M. D. Settle, T. F. Krauss, Dependence of extrinsic loss on group velocity in photonic crystal waveguides, Opt. Express 15: 13129-13138, 2007. 